\title{
Action of Cleistanthins A and B on Alpha Adrenoceptors in Rats
}

\author{
Mageshwaran Lakshmanan', Zachariah Bobby' ${ }^{2}$, Raveendran Ramasamy ${ }^{3 *}$ \\ 'Department of Pharmacology, SMMCH \& RI, Mangadu, Chennai-69, Tamilnadu, INDIA. \\ 2Department of Biochemistry, Jawaharlal Institute of Postgraduate Medical Education and Research (JIPMER), Puducherry, INDIA. \\ ${ }^{3}$ Professor, Department of Pharmacology, Jawaharlal Institute of Postgraduate Medical Education and Research, Puducherry, INDIA.
}

\begin{abstract}
Objectives: Cleistanthins $A$ and $B(C A B)$, active phytochemicals present in Cleistanthus collinus, have been proven to have alpha 1-adrenoceptor blocking property. However action on alpha 2-adrenoceptor is not studied. We aimed to study the action of CAB on alpha 2-adrenoceptor by measuring platelet aggregation, insulin and blood glucose levels with yohimbine as a standard comparator control and alpha 1-adrenoceptor by measuring the blockade of relaxation induced by phenylephrine in the jejunum of Wistar albino rats. Materials and methods: Forty eight adult male Wistar rats were divided into eight drug groups of six each, namely control (CMC $0.5 \%$ ), yohimbine $2 \mathrm{mg} / \mathrm{kg}, 10,30$ and $100 \mathrm{mg} / \mathrm{kg}$ doses of cleistanthin A and cleistanthin B. Blood samples were drawn at baseline, after $5 \mathrm{~h}$ and after high-epinephrine dose $(5 \mathrm{mg} / \mathrm{kg}$ ) from the retro-orbital sinus and used for estimation of platelet aggregation and plasma insulin levels. Blood glucose levels were estimated at six different time points. Degree of jejunal relaxation was studied by relaxing the acetylcholine pre-contracted jejunum with phenylephrine. Results: All groups, except control and cleistanthin A $10 \mathrm{mg} / \mathrm{kg}$ group, significantly inhibited the platelet aggregation when compared to the respective baseline values $(p<0.05)$. No significant difference was observed between different drug groups in plasma insulin and blood glucose levels. With regard to phenylephrine induced jejunal relaxation, the median $\mathrm{IC}_{50}$ for all three doses of cleistanthin $\mathrm{A}$ and cleistanthin B $100 \mathrm{mg} / \mathrm{kg}$ differ significantly from that of control (CMC 0.5\%). Conclusion: $\mathrm{CAB}$ block both alpha 1 and 2-adrenoceptorand hence are non-selective alpha-adrenoceptor blockers.
\end{abstract}

Key words: Cleistanthus Collinus, Cleistanthin A, Cleistanthin B, Alpha-adrenoceptor, Rat, Platelet aggregation.

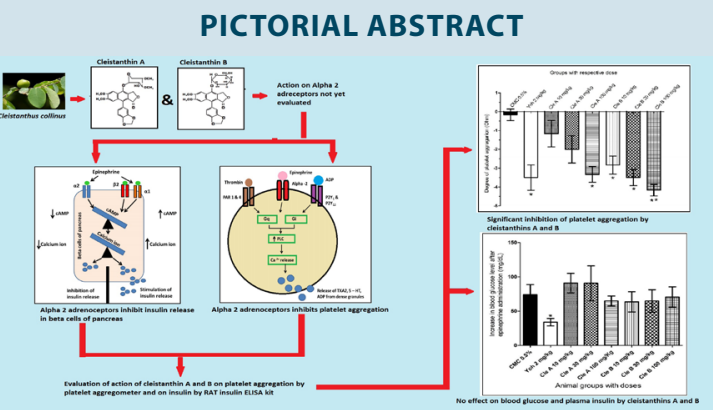

Correspondence :

Dr. Raveendran Ramasamy,

Professor, Department of Pharmacology, Jawaharlal Institute of Postgraduate Medical Education and Research (JIPMER), Puducherry, INDIA.

Phone no: 0413-2273134

E-mail: dr.ravee@gmail.com

DOI: 10.5530/jyp.2016.3.3

\section{INTRODUCTION}

Consumption of toxic plants is one of the most common modes of suicide. The shrub Cleistanthus collinus is a toxic plant commonly seen in South East Asian countries and abused as suicidal poison. ${ }^{1}$ Patients with Cleistanthus collinus poisoning present with initial symptoms of diarrhoea, vomiting and leg cramps progressing to hypokalaemia, metabolic acidosis and hypotension. ${ }^{2,3}$ The hypotension developed is resistant to standard pressor agents like dopamine and noradrenaline. ${ }^{4}$ It was hypothesized that pressor resistant hypotension induced by the poisoning might be due to blockade of alpha adrenoceptors by the active phytochemicals. A study with crude extracts from the leaves of Cleistanthus collinus showed the relaxing effect on isolated guinea pig aorta suggesting that the active phytochemicals present in the crude extract block the alpha adrenoceptors. ${ }^{5}$

Cleistanthins A and B are the two main active phytochemicals present in the most of the aerial parts of the plants. ${ }^{6}$ Subsequent study with cleistanthin A and B on guinea pig vas deferens confirmed the blockade of phenylephrine induced contractions. ${ }^{7}$ Phenylephrine shows selective agonism to alpha 1-adrenoceptor and the fact that cleistanthins A and B block the contractions induced by phenylephrine indicates that cleistanthins $\mathrm{A}$ and $\mathrm{B}$ have alpha 1-adrenoceptor blocking property.

However, to the best of our knowledge, the specificity of the compounds with respect to alpha 1 and 2-adrenoceptors has not been reported. The action of cleistanthins $\mathrm{A}$ and $\mathrm{B}$ against specific alpha 2-adrenoceptor agonists and on alpha 1-adrenoceptor induced relaxation of intestine are not found in the literature. Therefore, it is not known whether cleistanthins A and B block only the alpha 1-adrenoceptors or both alpha 1 and 2-adrenoceptors. The alpha 2-adrenoceptors are located post junctionally in platelets, brain, beta cells of the pancreas and blood vessels. Stimulation of alpha 2-adrenoceptors causes platelet aggregation and inhibition of insulin release. ${ }^{8}$ Thus, our work was intended to explore the action of cleistanthins A and B on (a) alpha 2-adrenoceptors by studying their effect on platelet aggregation and insulin release, and (b) alpha 1-adrenoceptors using phenylephrine induced jejunal relaxation in adult male Wistar albino rats.

\section{MATERIALS AND METHODS}

\section{The plant material}

The botanical name of the plant is Cleistanthus collinus (Roxb.) Benth.ex Hook.f belonging to the genus 'Cleistanthus' under the family 'Phyllanthaceae. The identity number for this species given by 'The international plant name index' is 341328-1. The leaves from this plant were collected during February to April of every year in Pondicherry and Cuddalore districts of Tamil Nadu, India. They were identified and certified by the Botanical Survey of India (BSI), Coimbatore (BSI/SC/5/23/08-09/ Tech.241). A voucher specimen of the plant is kept in the Department of Pharmacology, Jawaharlal Institute of Postgraduate Medical Education and Research (JIPMER), for further reference. 


\section{Ethics and Animals}

The study was approved by the Institute Animal Ethics Committee, JIPMER, Puducherry. Forty eight healthy, adult, male Wistar albino rats weighing $\geq 200$ g were used for the study. All the rats were obtained from the Central Animal House, JIPMER. The rats were allowed to adapt to the laboratory conditions for a week and were maintained as per standard requirements (spacious housing cages, $12 \mathrm{~h}$ dark-light cycle and $24 \pm 4^{\circ} \mathrm{C}$ temperature) throughout the study period. Rats were fed with standard rodent pellet supplied by Amrut laboratory feeds, Sangli, India. Water was provided ad libitum.

\section{Extracts and Chemicals}

Cleistanthin A and B are extracted from the dried leaves by cold extraction method as specified in the previous work. ${ }^{9}$ Since cleistanthins A and B are insoluble in water, carboxymethylcellulose (CMC) $0.5 \%$ was used as a solvent and a suspension was prepared. Yohimbine, epinephrine and phenylephrine pure powder were obtained from Sigma -Aldrich, Missouri, USA. Stock solutions were prepared with distilled water.

\section{Tyrode solution}

For preparing three litres of tyrode solution, by using an electronic balance, $24 \mathrm{~g}$ of sodium chloride, $6 \mathrm{~g}$ of anhydrous dextrose, $3 \mathrm{~g}$ of sodium bicarbonate, $600 \mathrm{mg}$ of potassium chloride, $300 \mathrm{mg}$ of magnesium chloride and $150 \mathrm{mg}$ of sodium dihydrogen phosphate monohydrate were added in deionized water and mixed thoroughly. To this solution, $600 \mathrm{mg}$ of anhydrous calcium chloride was added slowly with constant mixing of the solution.

\section{Reagents}

Lyophilized epinephrine and native type 1 collagen fibrils used for platelet aggregation were obtained from Chrono Log Corporation, Pennsylvania, USA. The reagents used for insulin estimation were obtained from Crystal Chem, Illinois, USA. Others chemicals and materials used for anaesthesia, blood collection and processing techniques were obtained from Mercks chemicals, India.

\section{Working procedure}

Forty eight rats were divided into eight different drug groups of six animals each. The control group received CMC $0.5 \%$ and the comparator group received yohimbine $2 \mathrm{mg} / \mathrm{kg}$. Other six groups received 10,30 and $100 \mathrm{mg} / \mathrm{kg}$ doses of cleistanthin A and similar doses of cleistanthin B. All the rats were kept under fasting for twelve hours before blood collection for the baseline data. The working procedure is shown in Figure 1.

Under general anaesthesia $0.75 \mathrm{ml}$ of blood was collected from the retroorbital plexus and used for the estimation of baseline blood glucose, plasma insulin level and platelet aggregation. The drug of respective group was administered by gavage and care was taken not to exceed the volume of $0.3 \mathrm{ml}$. A drop of blood from rat tail vein was collected at $30 \mathrm{~min}, 1.5 \mathrm{~h}$ and $3 \mathrm{~h}$. At the end of $5 \mathrm{~h}, 0.75 \mathrm{ml}$ of blood was collected from retroorbital plexus and used for estimation of blood glucose, plasma insulin and platelet aggregation. Subcutaneous administration of epinephrine at a dose of $5 \mathrm{mg} / \mathrm{kg}$ (high-dose epinephrine) was given and blood glucose and plasma insulin estimation were done.

\section{Estimation of blood glucose}

Blood glucose was estimated using a glucometer (Acon laboratories Inc, California, USA) with a drop of blood collected from retro-orbital plexus at baseline, at the end of $5 \mathrm{~h}$ and after high-dose epinephrine and from rat tail vein at $30 \mathrm{~min}, 1.5 \mathrm{~h}$ and $3 \mathrm{~h}$.

\section{Estimation of plasma insulin levels}

The level of plasma insulin was estimated using ultra-sensitive rat insulin ELISA kit (Crystal chem, Illinois, USA). Multi-analyzer Infinite M 200
TECAN (Tecan group Ltd, Maennedorf, Switzerland) with Magellan software (Version 6.5, Tecan group Ltd, Maennedorf, Switzerland) was used to calculate the absorbance. Blood samples of $0.25 \mathrm{ml}$ with $5 \mu \mathrm{L}$ of $0.018 \%$ dipotassium EDTA as anticoagulant was centrifuged at $4^{\circ} \mathrm{C}$ for $20 \mathrm{~min}$ at $2000 \mathrm{rpm}$ using a cooling centrifuge. The plasma was then separated, collected in Eppendorf tube and stored at $-80^{\circ} \mathrm{C}$. All the reagents and plasma samples were thawed and brought to room temperature on the day of estimation. To each well, $95 \mu \mathrm{L}$ of sample diluent was dispensed and $5 \mu \mathrm{L}$ of sample and standards were added into the respective wells and incubated for $2 \mathrm{~h}$ at $4^{\circ} \mathrm{C}$. Using automated aspirator, all the wells were washed for five times with $300 \mu \mathrm{L}$ of wash buffer. After this, $100 \mu \mathrm{L}$ of reconstituted anti-insulin enzyme conjugate was added to all the wells and incubated at the room temperature for $30 \mathrm{~min}$.

After $30 \mathrm{~min}$, the wells were washed with $300 \mu \mathrm{L}$ of wash buffer for seven times and excess solution was removed. Then $100 \mu \mathrm{L}$ of enzyme substrate (3, 3', 5, 5'-tetramethylbenzidine) was added quickly to each well and the microplate was kept in a dark room for $40 \mathrm{~min}$ at room temperature. Violet colour was seen after 40 min which turned to yellow after the addition of enzyme reaction stop solution ( $1 \mathrm{~N}$ sulphuric acid) $100 \mu \mathrm{L}$ to each well. Using a multi-analyser, the absorbance at $450 \mathrm{~nm}$ (A450) and $630 \mathrm{~nm}$ (A630) were calculated and the difference (A450-630) was estimated. The standard graph was plotted by Magellan software using the standard insulin concentrations and insulin level in samples were calculated by interpolation method.

\section{Estimation of degree of platelet aggregation}

The degree of platelet aggregation was analysed using lumi-aggregometer model 700 with Agrolink software (Chrono-Log Corporation, Pennsylvania, USA). The whole blood of $0.45 \mathrm{ml}$ volume was mixed with $50 \mu \mathrm{L}$ of $3.8 \%$ trisodium citrate anti-coagulant in a polycarbonate cuvette and was then made up to $1 \mathrm{ml}$ by adding pre-warmed $0.5 \mathrm{ml}$ of $0.9 \%$ saline and incubated at $37^{\circ} \mathrm{C}$ for $15 \mathrm{~min}$. After this, sample was kept inside the platelet aggregometer and electric rod was immersed. The temperature was maintained at $37^{\circ} \mathrm{C}$ with constant magnetic stir bar speed of $1200 \mathrm{rpm}$. Baseline impedance was noted down and $10 \mu \mathrm{L}$ of native collagen fibrils (type 1) from equine tendon suspended in isotonic solution at $\mathrm{pH}$ 2.7 and $1 \mu \mathrm{L}$ of $1 \mathrm{mM}$ epinephrine bitartrate were added quickly to the sample. The increase in resistance due to platelet activation and aggregation were displayed as impedance in ohm. Each sample was run for $6 \mathrm{~min}$ and the final value of impedance in ohm was noted down.

\section{Estimation of phenylephrine induced jejunal relaxation}

Data acquisition system attached with organ bath (Biopac systems Inc, California, USA) was used for estimation of phenylephrine induced jejunal relaxation. After the blood sample collection for biochemical parameters, the rat was sacrificed by cervical dislocation and abdomen was opened. Caecum was identified and jejunum was traced back from caecum. Around $5 \mathrm{~cm}$ of jejunum along with mesentery was cut and immediately transferred to pre-warmed tyrode solution in a petridish which was constantly aerated. Around $3 \mathrm{~cm}$ of jejunum was cut and the both ends of the jejunum were tied with thread. The organ bath was loaded with tyrode solution which was maintained at $37 \pm 1^{\circ} \mathrm{C}$. The jejunum was then mounted in the constantly aerated tissue chamber of the organ bath and was allowed to relax for $20 \mathrm{~min}$. Tyrode solution was changed four times and aeration was maintained at 1 bubble per second. After $20 \mathrm{~min}$, the jejunum was pre-contracted to the maximum by adding acetylcholine in the cumulative graded dose manner. The degree of contraction was recorded by the data acquisition system and was displayed as the height of the curve. After attaining the maximal contraction with acetylcholine, phenylephrine was added in the graded doses (Acetylcholine from $1 \mathrm{mcg} / \mathrm{ml}$ to $128 \mathrm{mcg} / \mathrm{ml}$ and phenylephrine from $1 \mathrm{mcg} / \mathrm{ml}$ to $1500 \mathrm{mcg} / \mathrm{ml}$ ). The degree of relaxation by phenylephrine 


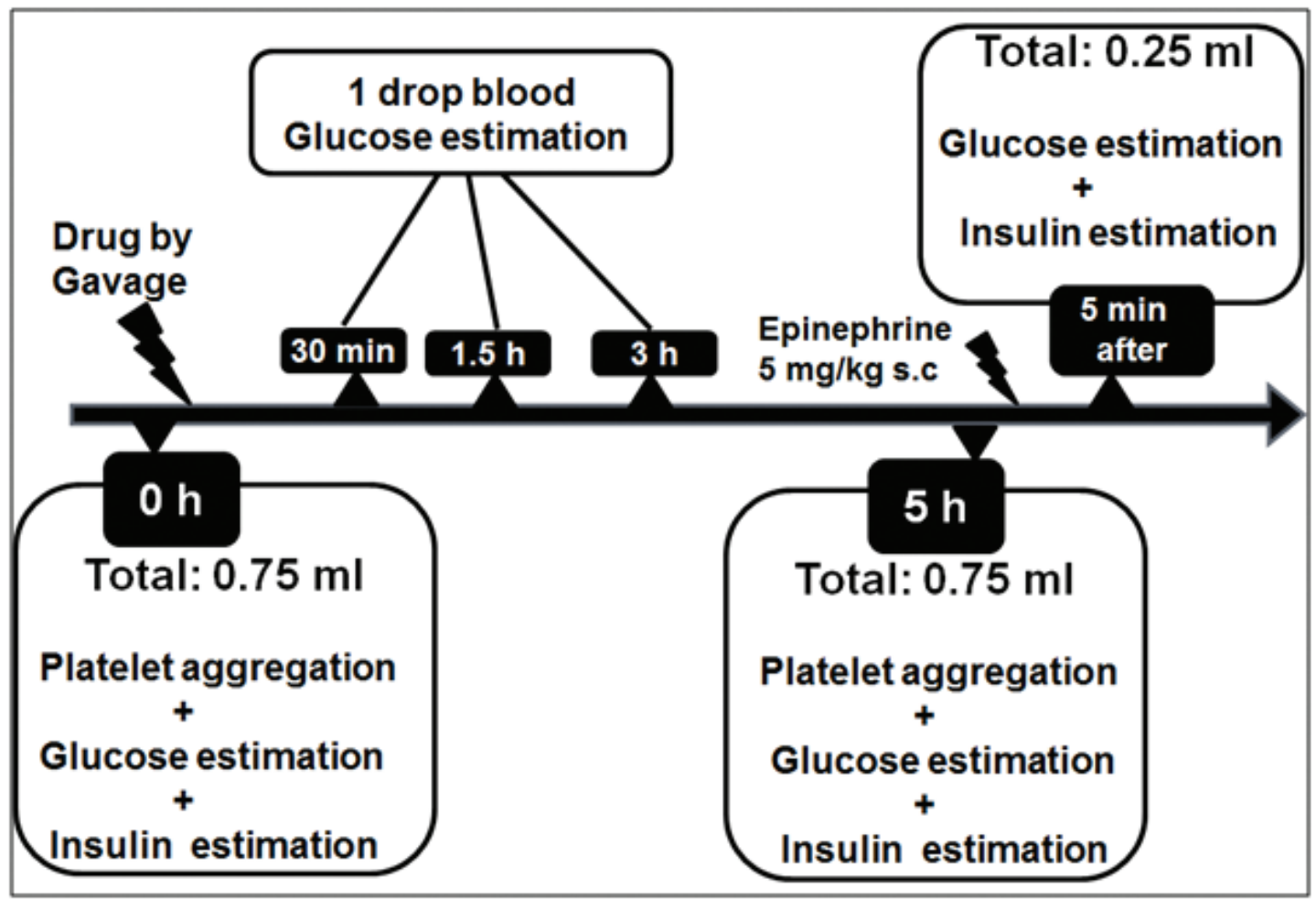

Figure 1: Working procedure. $h=$ hour; $\min =$ minute; s.c=subcutaneous administration.

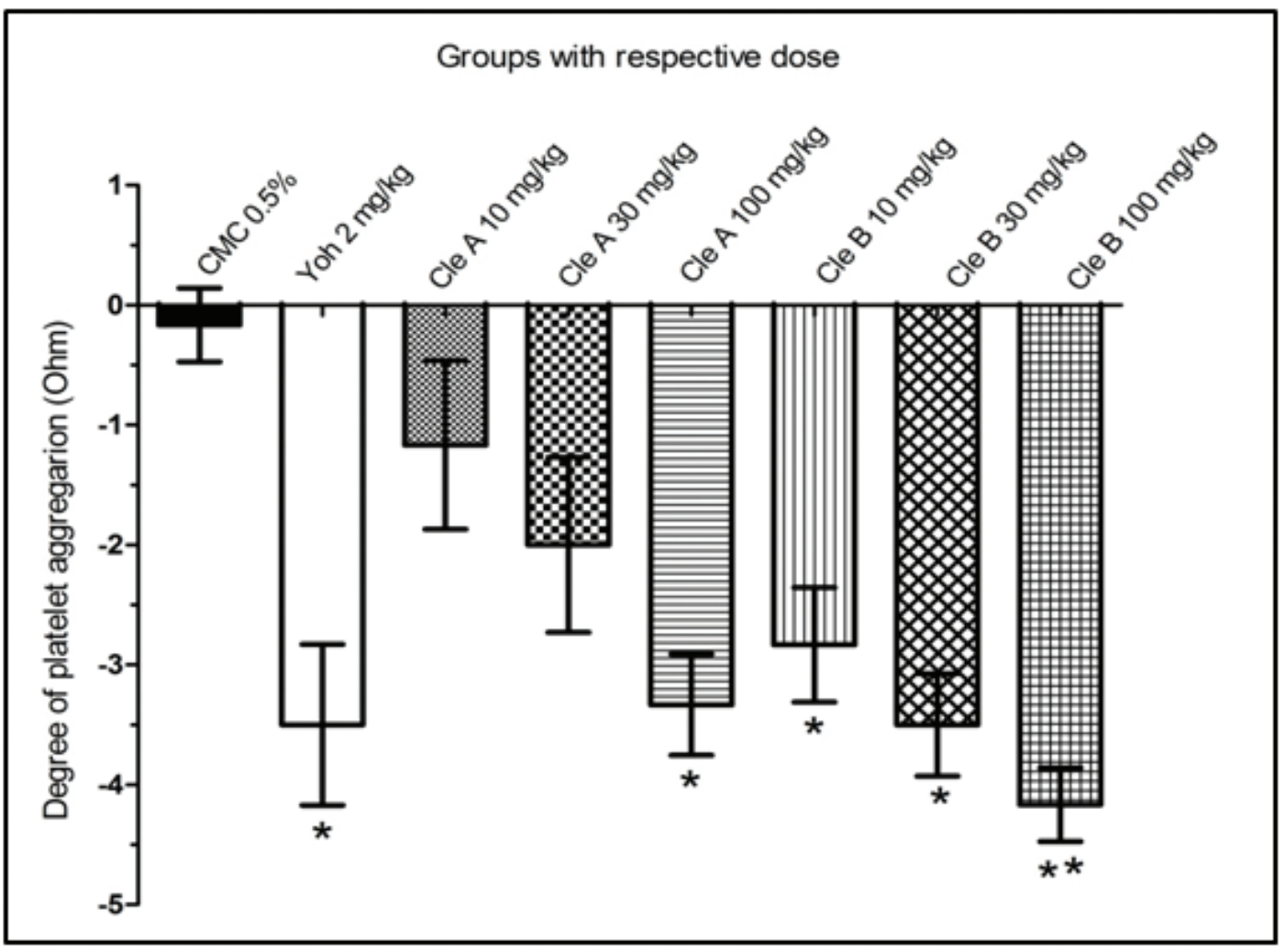

Figure 2: Bar diagram showing the reduction in degree of platelet aggregation after $5 \mathrm{~h}$ from baseline in the presence of threshold collagen (10 $\mu \mathrm{g} / \mathrm{ml})$ and epinephrine (1 mM) by various doses of cleistanthins $A$ and $B$ in Wistar albino rats. $\mathrm{N}=6$ animals/ group. Data are expressed as mean (SEM). * $=p<0.05$ and ${ }^{* *}$ $=p<0.001$ when compared to CMC $0.5 \%$ (control) group. One-way ANOVA followed by Bonferroni's multiple comparison was used to test the significant difference. Cle=Cleistanthin; $C M C=$ Carboxymethylcellulose; Yoh=Yohimbine. 


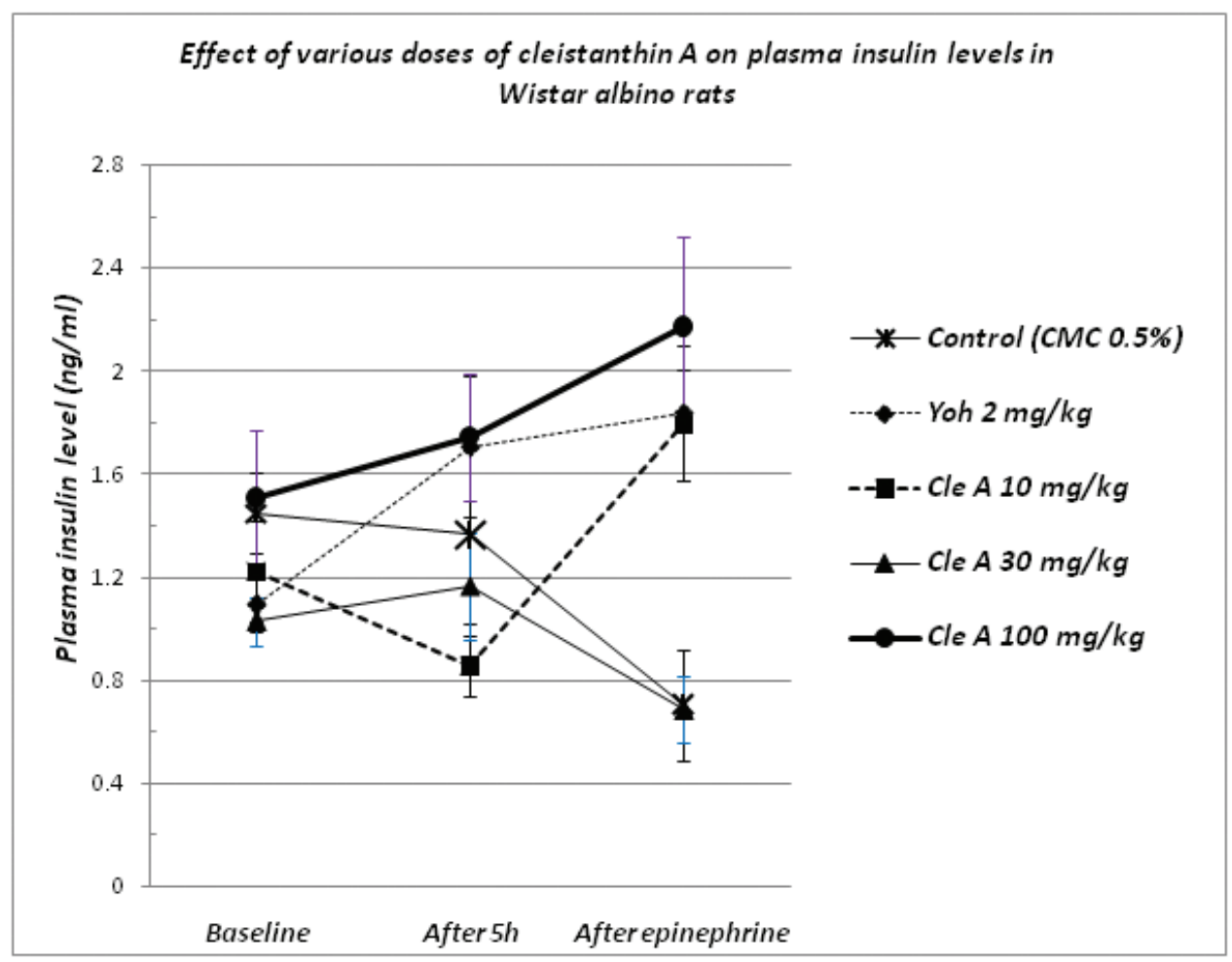

Figure 3: Line diagram showing the effect of various doses of cleistanthin A on plasma insulin levels at baseline, after $5 \mathrm{~h}$ and after subcutaneous epinephrine $(5 \mathrm{mg} / \mathrm{kg}$ ) injection in Wistar albino rats. Data are expressed as mean (SEM). $\mathrm{N}=6$ animals/group. Two-way repeated measures ANOVA followed by Bonferroni's multiple comparison was performed and no significant difference was observed between the groups. $\mathrm{Cle}=\mathrm{Cleistanthin} ; \mathrm{CMC}=\mathrm{Carboxymethylcellulose} \mathrm{h}=\mathrm{hour}$; Yoh=Yohimbine.

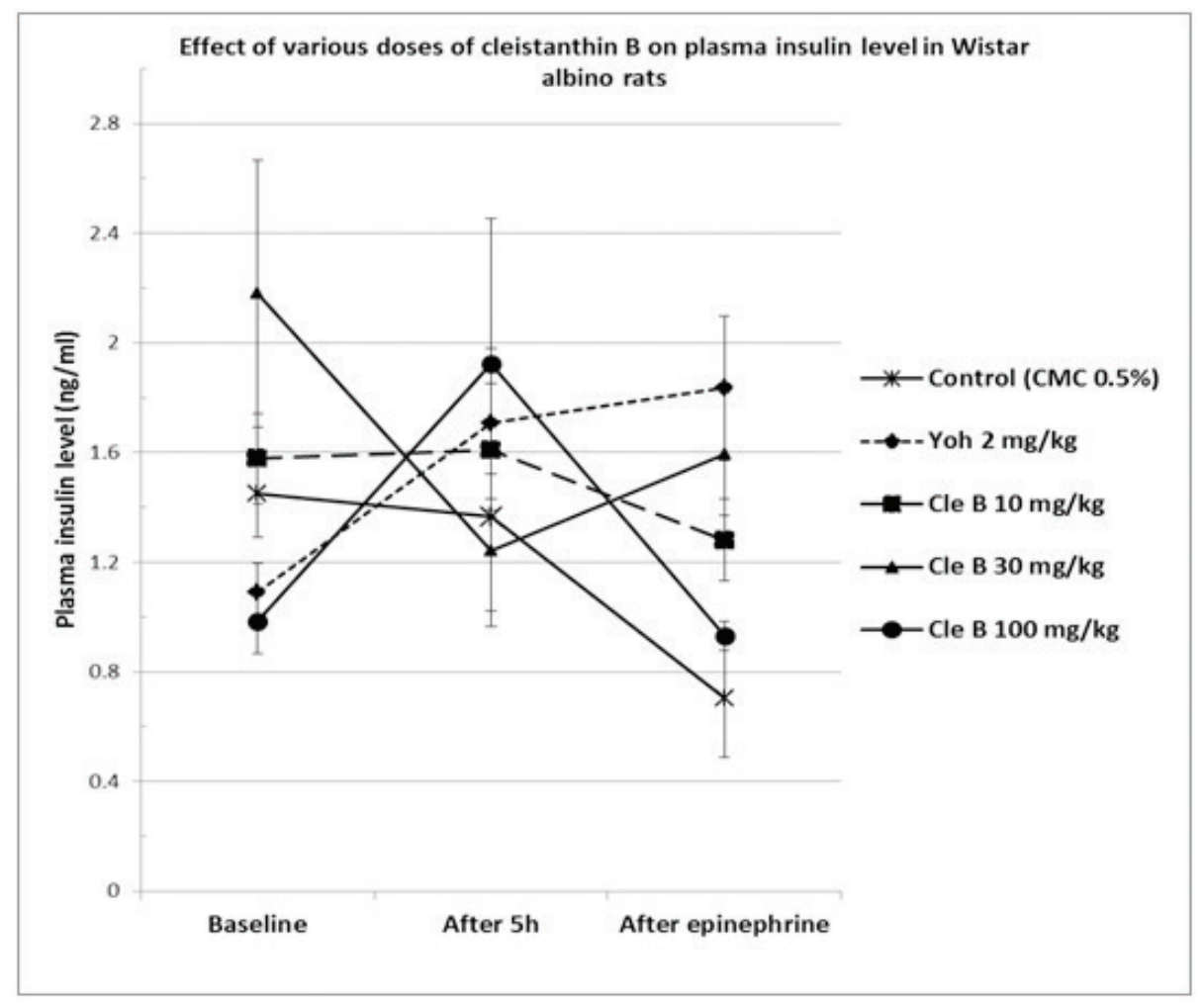

Figure 4: Line diagram showing the effect of various doses of cleistanthin B on plasma insulin levels at baseline, after $5 \mathrm{~h}$ and after subcutaneous epinephrine $(5 \mathrm{mg} / \mathrm{kg}$ ) injection in Wistar albino rats. Data are expressed as mean (SEM). $\mathrm{N}=6$ animals/group. Two-way repeated measures ANOVA followed by Bonferroni's multiple comparisons was performed and no significant difference was observed between the groups. $\mathrm{Cle}=\mathrm{Cleistanthin} ; \mathrm{CMC}=\mathrm{Carboxymethylcellulose;} \mathrm{h}=\mathrm{hour}$; Yoh=Yohimbine. 


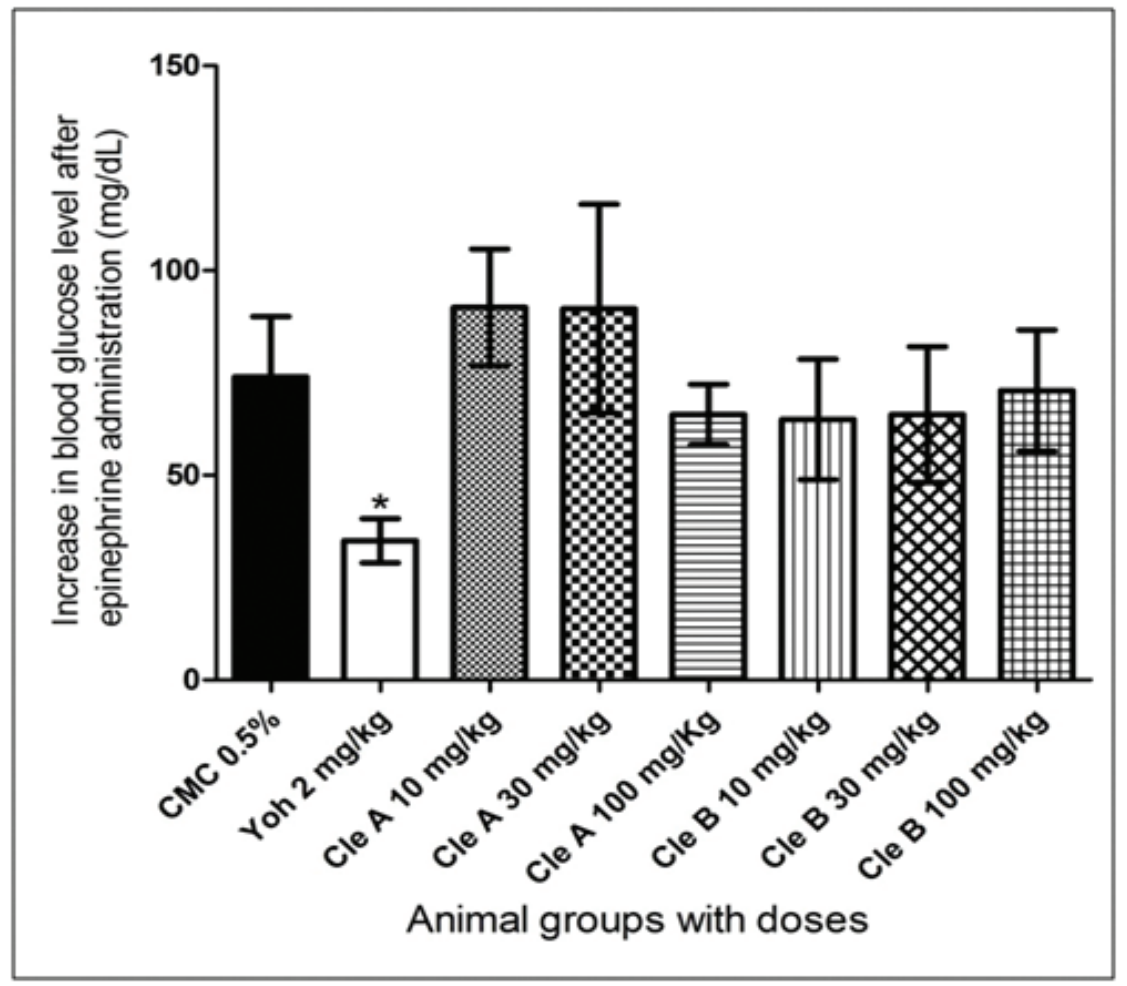

Figure 5: Bar diagram showing the effect of various doses of cleistanthins A and B on elevation of blood glucose level (in mg/dl) after subcutaneous administration of high dose epinephrine ( $5 \mathrm{mg} / \mathrm{kg}$ ) in Wistar albino rats. $\mathrm{N}=6$ animals/group. Data are mean (SEM). ${ }^{*} \mathrm{p}<0.05$ when compared to control (CMC $\left.0.5 \%\right)$. One-way ANOVA followed by Bonferroni's multiple comparison was used. CMC=Carboxymethylcellulose; Yoh=Yohimbine; Cle=Cleistanthin.

from the maximal contraction was recorded. The maximum height of the curve from the baseline (representing the maximum contraction-Y) was noted. Relaxation obtained for each graded dose of phenylephrine (X) was calculated from the line of maximum height. The percentage of relaxation $\left(\mathrm{X} / \mathrm{Y}^{\star} 100\right)$ was calculated and the inhibitory concentration 50 $\left(\mathrm{IC}_{50}\right)$ for each group was calculated.

\section{Statistical analyses}

Statistical analyses were done using Graphpad Prism version 5 software. Baseline difference between the groups in the degree of platelet aggregation, blood glucose, and insulin levels were analyzed using one-way ANOVA. Degree of platelet aggregation compared to baseline within the group was analysed using paired t test. Mean reduction in platelet aggregation between the groups was analyzed using one-way ANOVA followed by Bonferroni's multiple comparison post-hoc test. Difference in plasma insulin levels and blood glucose in different time points between different groups were analyzed by two-way repeated measures ANOVA. $\mathrm{IC}_{50}$ for jejunal relaxation between different drug groups was analyzed using Kruskal Wallis test followed by Dunn's multiple comparison post-hoc test. $\mathrm{P}<0.05$ was considered statistically significant.

\section{RESULTS}

\section{Baseline characteristics}

The baseline characteristics of degree of platelet aggregation, blood glucose and plasma insulin levels are summarized in Table 1. No significant difference was observed between the groups.

\section{Effect of cleistanthins $A$ and $B$ on platelet aggregation}

The degree of platelet aggregation of different groups at baseline and after $5 \mathrm{~h}$ of drug administration is summarized in Table 2 and the mean reduction in degree of platelet aggregation after $5 \mathrm{~h}$ from baseline is shown in Figure 2. Except control (CMC 0.5\%) and cleistanthin A $10 \mathrm{mg} / \mathrm{kg}$ group, all groups significantly inhibited platelet aggregation when compared to the respective baseline values. The mean reduction in degree of platelet aggregation for the cleistanthin B $30 \mathrm{mg} / \mathrm{kg}$ group was $3.5 \mathrm{ohm}$ and was equal to that of yohimbine $(2 \mathrm{mg} / \mathrm{kg})$ group and the mean reduction in platelet aggregation of cleistanthin B $100 \mathrm{mg} / \mathrm{kg}$ group is $4.16 \mathrm{ohm}$ which exceeded that produced by the yohimbine group. Yohimbine $2 \mathrm{mg} / \mathrm{kg}$, cleistanthin A $100 \mathrm{mg} / \mathrm{kg}$ and all three doses of cleistanthin B (10 mg/kg, $30 \mathrm{mg} / \mathrm{kg}$ and $100 \mathrm{mg} / \mathrm{kg})$ were found to be significantly differing from the control (CMC $0.5 \%)$ in inhibiting platelet aggregation.

\section{Effect of cleistanthins $A$ and $B$ on plasma insulin levels}

The plasma insulin levels measured at baseline, after $5 \mathrm{~h}$ and after subcutaneous administration of epinephrine $5 \mathrm{mg} / \mathrm{kg}$ of different drug group in $\mathrm{ng} / \mathrm{ml}$ are shown in Figure 3 and 4 . The plasma insulin levels in the control (CMC 0.5\%) group was reduced from $1.45 \mathrm{ng} / \mathrm{ml}$ to $1.37 \mathrm{ng} / \mathrm{ml}$ after $5 \mathrm{~h}$ and decreased further to $0.7 \mathrm{ng} / \mathrm{ml}$ after epinephrine $5 \mathrm{mg} / \mathrm{kg}$ administration. Yohimbine $2 \mathrm{mg} / \mathrm{kg}$ increased the plasma insulin level from $1.09 \mathrm{ng} / \mathrm{ml}$ to $1.71 \mathrm{ng} / \mathrm{ml}$ after $5 \mathrm{~h}$ and a minimal rise $(1.83 \mathrm{ng} / \mathrm{ml})$ was observed after epinephrine $5 \mathrm{mg} / \mathrm{kg}$ administration. However, no significant difference was observed between different drug groups when the mean change in plasma insulin levels from 'baseline' to ' $5 \mathrm{~h}$ ' and from ' 5 h' to 'after-epinephrine' were compared.

\section{Effect of cleistanthins A and B on blood glucose level}

The percentage change in blood glucose from the baseline at five different time points in different drug groups were calculated and shown in Table 3. No significant difference was observed. The increase in blood glucose level after subcutaneous administration of epinephrine $5 \mathrm{mg} / \mathrm{kg}$ 
LAKSHMANAN et al.: Action of cleistanthins A and B on alpha adrenoceptors in rats

\begin{tabular}{cccc} 
Table 1: Baseline characteristics of various parameters in different drug groups in Wistar albino rats \\
\hline Group name (dose) & $\begin{array}{c}\text { Degree of Platelet } \\
\text { aggregation } \\
\text { [Ohm] }\end{array}$ & $\begin{array}{c}\text { Plasma Insulin } \\
\text { [ng/ml] }\end{array}$ & $\begin{array}{c}\text { Blood glucose } \\
\text { [mg/dl] }\end{array}$ \\
\hline Control (CMC 0.5\%) & $4.83(0.75)$ & $1.45(0.38)$ & $82.17(14.37)$ \\
Yohimbine (2 mg/kg) & $4.33(1.86)$ & $1.09(0.26)$ & $95(6.48)$ \\
Cleistanthin A (10 mg/kg) & $5.33(1.21)$ & $1.22(0.48)$ & $71.33(7.47)$ \\
Cleistanthin A (30 mg/kg) & $5.16(1.47)$ & $1.03(0.23)$ & $86(18.13)$ \\
Cleistanthin A (100 mg/kg) & $5.16(1.32)$ & $1.5(0.65)$ & $76.17(20.59)$ \\
Cleistanthin B (10 mg/kg) & $5.16(0.75)$ & $1.58(0.4)$ & $73.5(10.56)$ \\
Cleistanthin B (30 mg/kg) & $5.5(1.04)$ & $1.18(1.2)$ & $85.67(4.27)$ \\
Cleistanthin B (100 mg/kg) & $5.83(0.75)$ & $0.98(0.29)$ & $81.17(11.85)$ \\
F Value & 0.816 & 0.959 & 2.15 \\
P value & 0.579 & 0.473 & 0.06 \\
\hline
\end{tabular}

Data are expressed as mean (SD); $\mathrm{N}=6$ animals/ group; One-way ANOVA was performed for each parameter and no significant difference was observed between the groups. $\mathrm{CMC}=$ Carboxymethylcellulose.

Table 2: Effect of various doses cleistanthins A and B on the degree of platelet aggregation in the presence of threshold collagen $(10 \mu \mathrm{g} / \mathrm{ml})$ and epinephrine $(1 \mathrm{mM})$ in Wistar albino rats

\begin{tabular}{ccccc}
\hline \multirow{2}{*}{ Group name (dose) } & \multicolumn{3}{c}{ Degree of platelet aggregation (Ohm) } & \multirow{2}{*}{ P value } \\
\cline { 2 - 4 } & Baseline & After $5 \mathrm{~h}$ & Mean Reduction [Cl] & \\
\hline Control (CMC 0.5\%) & $4.83(0.75)$ & $4.66(1.03)$ & $0.16[-0.95$ to 0.62$]$ & $0.61(\mathrm{NS})$ \\
Yohimbine $(2 \mathrm{mg} / \mathrm{kg})$ & $4.33(1.86)$ & $0.83(0.75)$ & $3.5[1.77$ to 5.22$]$ & 0.003 \\
Cleistanthin A (10 mg/kg) & $5.33(1.21)$ & $4.16(1.32)$ & $1.16[-0.64$ to 2.97$]$ & $0.158(\mathrm{NS})$ \\
Cleistanthin A (30 mg/kg) & $5.16(1.47)$ & $3.16(2.71)$ & $2[0.12$ to 3.87$]$ & 0.04 \\
Cleistanthin A (100 mg/kg) & $5.16(1.32)$ & $1.83(1.47)$ & $3.33[2.24$ to 4.41$]$ & $<0.001$ \\
Cleistanthin B (10 mg/kg) & $5.16(0.75)$ & $2.33(1.03)$ & $2.83[1.61$ to 4.06$]$ & 0.002 \\
Cleistanthin B (30 mg/kg) & $5.5(1.04)$ & $2(0.63)$ & $3.5[2.4$ to 4.6$]$ & $<0.001$ \\
Cleistanthin B (100 mg/kg) & $5.83(0.75)$ & $1.66(1.21)$ & $4.16[3.38$ to 4.95$]$ & $<0.001$ \\
\hline
\end{tabular}

Data are expressed in mean (SD) and effect change as mean [CI]. N=6 animals/group; Two tailed paired $t$ test was performed to test significant difference. $\mathrm{CI}=95 \%$ Confidence interval; $\mathrm{CMC}=$ Carboxymethylcellulose; NS=Not significant.

Table 3: Effect of various doses of cleistanthins A and B on blood glucose levels in Wistar albino rats

\begin{tabular}{|c|c|c|c|c|c|}
\hline \multirow[b]{2}{*}{ Group name (Dose) } & \multirow{2}{*}{$\begin{array}{l}\text { Baseline blood } \\
\text { glucose (mg/dl) }\end{array}$} & \multicolumn{4}{|c|}{ Percentage change (\%) in blood glucose levels from baseline } \\
\hline & & $\begin{array}{c}\text { After } 30 \mathrm{~min} \text { of } \\
\text { TADA }\end{array}$ & $\begin{array}{c}\text { After } 90 \mathrm{~min} \text { of } \\
\text { TADA }\end{array}$ & $\begin{array}{c}\text { After } 180 \mathrm{~min} \text { of } \\
\text { TADA }\end{array}$ & $\begin{array}{c}\text { After } 300 \mathrm{~min} \text { of } \\
\text { TADA }\end{array}$ \\
\hline Control (CMC 0.5\%) & $82.17(5.87)$ & $6.78(7.99)$ & $-2.23(5.73)$ & $3.5(6.85)$ & $21.86(11.31)$ \\
\hline Yohimbine (2 mg/kg) & $95(2.65)$ & $15.57(2.13)$ & $2.06(6.78)$ & $10.97(6.88)$ & $12.32(5.21)$ \\
\hline Cleistanthin A (10 mg/kg) & $71.33(3.05)$ & $18.72(14.78)$ & $16.87(11.22)$ & $16.12(12.27)$ & $8.98(10.28)$ \\
\hline Cleistanthin A (30 mg/kg) & $86(7.4)$ & $9.41(7.52)$ & $-3.55(9.34)$ & $12.7(11.55)$ & $15.25(11.04)$ \\
\hline Cleistanthin A (100 mg/kg) & $76.17(8.41)$ & $-2.18(10.25)$ & $16.77(11.7)$ & $19.67(23.03)$ & $20.64(18.09)$ \\
\hline Cleistanthin B (10 mg/kg) & $73.5(4.31)$ & $11.49(6.56)$ & $8.23(6.88)$ & $24.55(8.71)$ & $53.73(16.34)$ \\
\hline Cleistanthin B $(30 \mathrm{mg} / \mathrm{kg})$ & $85.67(1.76)$ & $6.61(9.13)$ & $10.4(8.85)$ & $13.07(13.24)$ & $18.09(5.36)$ \\
\hline Cleistanthin B (100 mg/kg) & $81.17(4.84)$ & $19.57(6.76)$ & $14.39(7.83)$ & $15.56(5.79)$ & $21.38(8.76)$ \\
\hline
\end{tabular}

Data are expressed as mean (SEM). N=6 animals/ group. Two-way repeated measures ANOVA with time and drug as factors was performed and no significant difference $(\mathrm{p}=0.388)$ was observed between the groups. $\mathrm{CMC}=$ Carboxymethylcellulose; TADA=Time after drug administration; NS=not significant. 


\begin{tabular}{|c|c|}
\hline Group name (Dose) & $\mathrm{IC}_{50}$ of phenylephrine response $(\mu \mathrm{g} / \mathrm{ml})$ \\
\hline Control (CMC 0.5\%) & $138.5[75.85,189.9]$ \\
\hline Yohimbine $(2 \mathrm{mg} / \mathrm{kg})$ & $179.1[-16.49,223]$ \\
\hline Cleistanthin A $(10 \mathrm{mg} / \mathrm{kg})$ & $644[419.4,774]^{*}$ \\
\hline Cleistanthin A $(30 \mathrm{mg} / \mathrm{kg})$ & $759.8[626.3,905.3]^{*, \#}$ \\
\hline Cleistanthin A (100 mg/kg) & $752.3[515.4,1828]^{*, *}$ \\
\hline Cleistanthin B (10 mg/kg) & $413[327.6,551.5]$ \\
\hline Cleistanthin B (30 mg/kg) & $289.8[125.1,659]$ \\
\hline Cleistanthin B $(100 \mathrm{mg} / \mathrm{kg})$ & $641.7[348.2,1293]^{*}$ \\
\hline
\end{tabular}

Data are median [Interquartile range]. $\mathrm{N}=6$ animals/group. Kruskal-Wallis test followed by Dunn's multiple comparison was performed. ${ }^{\star} \mathrm{p}<0.05$ when compared to control and \# $\mathrm{p}<0.05$ when compared to yohimbine $2 \mathrm{mg} / \mathrm{kg}$ group. $\mathrm{CMC}=$ Carboxymethylcellulose; IC=inhibitory concentration. IQ=Interquartile range.

in different drug group from $5 \mathrm{~h}$ value was measured and is shown in Figure 5. The level of increase in blood glucose of yohimbine $2 \mathrm{mg} / \mathrm{kg}$ group significantly differed from control (CMC 0.5\%) group $(74 \pm 36.19$ $\mathrm{mg} / \mathrm{dL}$ in control vs $34 \pm 13.16 \mathrm{mg} / \mathrm{dL}$ in yohimbine). Yohimbine significantly reduced the blood glucose elevation after epinephrine when compared to control group. None of the doses of cleistanthins A and B significantly altered the elevation of blood glucose after epinephrine administration when compared to control (CMC 0.5\%) group.

\section{Effect of cleistanthins $A$ and $B$ on phenylephrine induced jejunal relaxation}

The inhibitory concentration $50\left(\mathrm{IC}_{50}\right)$ for phenylephrine induced jejunal relaxation for different drug groups was calculated and is shown in Table 4. The median $\mathrm{IC}_{50}$ for all three doses of cleistanthin A $(10 \mathrm{mg} / \mathrm{kg}$, $30 \mathrm{mg} / \mathrm{kg}$, and $100 \mathrm{mg} / \mathrm{kg}$ ) and cleistanthin B $100 \mathrm{mg} / \mathrm{kg}$ differ significantly from the median $\mathrm{IC}_{50}$ of control (CMC 0.5\%). Moreover, it is evident that all three doses of cleistanthin A prevented phenylephrine induced relaxation while the cleistanthin B did only at the higher concentration $(100 \mathrm{mg} / \mathrm{kg})$.

\section{DISCUSSION}

The present study aimed at exploring the alpha 2-adrenoceptor blocking property of cleistanthins A and B by estimating platelet aggregation, plasma insulin and blood glucose levels and alpha 1-adrenoceptor blocking property by measuring the degree of relaxation of jejunum. We found that cleistanthins A and B inhibited platelet aggregation with a degree comparable to that of yohimbine, blocked phenylephrine from relaxing the rat jejunum and have no effect on plasma insulin levels and blood glucose levels.

The effect of cleistanthins A and B on platelet aggregation indicates that both the compounds have alpha 2-adrenoceptor blocking property. Platelets are populated with alpha 2-adrenoceptor and play a role similar to ADP and thrombin receptors in platelet aggregation. ${ }^{10}$ Any defect in alpha 2-adrenoceptors manifests clinically as haemostatic abnormalities and alteration in number or affinity of alpha 2-adrenoceptors in platelet membrane lead to defective platelet function. ${ }^{11}$ Under the threshold concentration of collagen with epinephrine as an agonist, the effect of many alpha 2-adrenoceptor antagonists on platelet aggregation has been explored. ${ }^{10,12-14}$

We used yohimbine (a standard alpha 2 adrenoceptor antagonist) as a comparator control and measured the degree of platelet aggregation at baseline and $5 \mathrm{~h}$ which was compared with cleistanthins $\mathrm{A}$ and B. Even though the half-life of yohimbine is $0.6 \mathrm{~h}$, it produces ten times more potent active metabolite called 11-OH yohimbine with a half-life of 6-7 h. ${ }^{15}$ Hence the effect of yohimbine could be observed even after $5 \mathrm{~h}$. In our study, yohimbine at $2 \mathrm{mg} / \mathrm{kg}$ dose has shown a significant inhibition of platelet aggregation after $5 \mathrm{~h}$ by blocking alpha 2 -adrenoceptor in platelet. Cleistanthin A at a dose of $100 \mathrm{mg} / \mathrm{kg}$ and cleistanthin B at all three doses produced a significant reduction in the degree of platelet aggregation similar to yohimbine when compared to control group. The fact that cleistanthins A and B produced significant inhibition of platelet aggregation comparable to yohimbine clearly indicates that the compounds have alpha 2-adrenoceptor blocking property. While cleistanthin B produced a significant reduction in platelet aggregation at all doses $(10,30$ and $100 \mathrm{mg} / \mathrm{kg}$ ), cleistanthin A did the same at $100 \mathrm{mg} / \mathrm{kg}$ dose only. The differential effect of cleistanthins A and B could be due to the difference in the potency between two compounds and hence 10 and $30 \mathrm{mg} / \mathrm{kg}$ of cleistanthin A were probably too low doses to produce a statistically significant effect.

No clear picture emerged from our data when we tried to delineate the effect of cleistanthins A and B on insulin and blood glucose levels. As the stimulation of alpha 2-adrenoceptors in beta cells of the pancreas decreases the insulin level, we used epinephrine, an alpha 2-adrenoceptor agonist to stimulate these receptors. Since alpha 2-adrenoceptor will be stimulated only at high concentration of epinephrine, $5 \mathrm{mg} / \mathrm{kg}$ of the same was used. Studies conducted on isolated beta cells of the pancreas demonstrated alpha 2-adrenoceptor blocking property by showing the increase, or at least no fall in insulin level after administration of epinephrine in the presence of alpha 2-adrenoceptor antagonists. ${ }^{16,17}$ Likewise, the increase in blood glucose level after high-dose epinephrine administration is reported to be blunted by alpha 2-adrenoceptor antagonists. ${ }^{18}$

In our study, we observed a fall in insulin levels after $5 \mathrm{~h}$ and a further decrease by high-dose epinephrine in control group due to fasting and epinephrine effect, respectively. These effects conform to normal physiology and are expected to happen in the control group. Even though the plasma insulin levels in yohimbine pre-treated animals appear to increase over time, the difference is not significant when compared to control. However, yohimbine significantly blunted the epinephrine-induced rise in blood glucose levels in comparison with control. Though yohimbine $2 \mathrm{mg} / \mathrm{kg}$ dose produced an expected effect on blood glucose level, its effect on plasma insulin levels could not achieve statistical significance. Probably a higher dose of yohimbine or the use of more number of animals would have made the difference.

Statistically, no effect was seen with cleistanthins A and B on plasma insulin and blood glucose levels. Even though both cleistanthins A and B have shown significant alpha 2-adrenoceptor blocking property on platelet, their effect on the beta cells of the pancreas could not be determined evidently. It is noteworthy to mention that even the comparator control (yohimbine) failed to produce a significant effect on plasma insulin levels in comparison with the control group.

This could be due to the fact that the present study is in vivo and multiple mechanisms are involved in controlling insulin release in in vivo. Blockade of presynaptic alpha 2-adrenoceptor of beta cells of pancreas can lead to a decrease in insulin level while blockade of alpha 2-adrenoceptor in alpha cells of the pancreas can lead to glucagon release. ${ }^{19}$ Glucagon in turn can stimulate insulin release. Moreover, insulin release induced by a rise in glucose level can also affect the final outcome. The complexity of receptor interactions leading to insulin release and the non-selectivity of yohimbine for alpha 2-adrenoceptor subtypes could be attributed to lack of significant difference between the yohimbine and control in terms of plasma insulin levels. A similar explanation for the failure of cleistanthins 
A and B to produce an effect on plasma insulin and blood glucose levels may be offered and applicable.

Rat jejunum is populated with both alpha 1 and beta 1 adrenoceptor subtypes and stimulation of either receptor will lead to jejunal relaxation..$^{20}$ We have studied the effect of cleistanthins A and B on phenylephrine induced jejunal relaxation. All doses of cleistanthin A and cleistanthin $B$ significantly prevented the phenylephrine induced jejunal relaxation. The $\mathrm{IC}_{50}$ values of all the doses of cleistanthin A and cleistanthin B $100 \mathrm{mg} / \mathrm{kg}$ differ significantly from the control group.

Phenylephrine being an alpha 1-adrenoceptor agonist relaxed the jejunum in the control group. Yohimbine being an alpha 2-adrenoceptor antagonist could not affect phenylephrine induced relaxation of rat jejunum. The fact that tissues in both control and yohimbine groups attained full relaxation by phenylephrine indicates that the effect is mediated by alpha 1-adrenoceptor. It was reported in a study conducted on isolated rat heart that both cleistanthins $\mathrm{A}$ and $\mathrm{B}$ had no effect on beta 1-adrenoceptors. ${ }^{7}$ This suggests that the blockade of phenylephrine induced relaxation could be due to alpha 1-adrenoceptor antagonism.

From the present study, it is evident that cleistanthins A and B effectively blocked the phenylephrine induced relaxation and this effect could be attributed to blockade of alpha 1-adrenoceptors. This finding is in agreement with the effect of cleistanthins $\mathrm{A}$ and $\mathrm{B}$ on rat vas deferens where both the compounds prevented the phenylephrine induced contractions. ${ }^{7}$

We have collected all the blood samples under general anaesthesia and any undue stress induced endogenous epinephrine release was avoided. We have estimated the plasma insulin levels using an ultra-sensitive rat ELISA kit that can detect insulin levels up to $\mathrm{ng} / \mathrm{ml}$ precise when compared to conventional radio-immuno assay technique.

Our study has a few limitations. Firstly, the plasma insulin levels were measured only at three different time points not six where blood glucose levels were estimated. This was to avoid exceeding the recommended volume of blood drawn per day from rat. This limitation prevented us from determining the trend in insulin levels and correlating the same with blood glucose levels. Secondly, the effect of cleistanthins A and B in jejunal relaxation could have been compared with that of a standard alpha 1-adrenoceptor antagonist such as prazosin. Nevertheless, it would not have affected the outcome of the study. We used a selective alpha 1-adrenoceptor agonist and also observed yohimbine treated rat jejunum attaining full relaxation. This indirectly, yet strongly suggests relaxation was mediated via alpha 1-adrenoceptor and hence cleistanthins A and B blocked the alpha 1-adrenoceptor.

\section{CONCLUSION}

In rats, cleistanthins $\mathrm{A}$ and $\mathrm{B}$ prevent the platelet aggregation and the extent of the effect is comparable to yohimbine. These compounds exert no effect on plasma insulin and blood glucose levels. Cleistanthins $A$ and $B$ block relaxation of jejunum induced by phenylephrine. Therefore Cleistanthins A and B block both alpha 1 and 2-adrenoceptors and hence are non-selective alpha adrenoceptor blockers.

\section{ACKNOWLEDGMENT}

The study was supported by the intramural fund from JIPMER institute, Puducherry, India.

\section{CONFLICT OF INTEREST}

The authors have no conflict of interests to declare.

\section{AUTHOR CONTRIBUTION}

The study was designed by the second and the third authors with contributions from the first author. The study was executed by the first author. All the authors have equal contribution in data analysis and writing up of the article.

\section{ABBREVIATIONS USED}

CAB: Cleistanthins A and B; CMC: Carboxymethylcellulose; Cle: Cleistanthin; JIPMER: Jawaharlal Institute of Postgraduate Medical Education and Research; Yoh: Yohimbine.

\section{ABOUT AUTHORS}

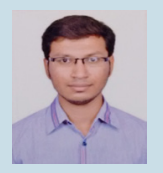

Dr. Mageshwaran Lakshmanan: Is an Assistant professor at the Department of Pharmacology, Srimuthukumaran medical college hospital and research institute (SMMCHRI), Chennai. His research interest is in the area of toxicology and pre-clinical evaluation of phytochemicals. He is also a member of the pharmacovigilance team in SMMCHRI.

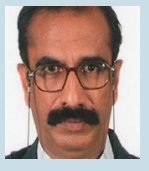

Dr. Raveendran Ramasamy: Is a professor in Department of Clinical Pharmacology, JIPMER, Pondicherry, India. He has more than 30 years of research and teaching experience. He has published more than 40 research papers apart from one book and software packages for computer assisted learning. His research interest is in the area of pre-clinical evaluation of phytochemicals, computer assisted learning (CAL) and receptor pharmacology.

\section{REFERENCES}

1. Bose A, Sejbaek CS, Suganthy P, Raghava V, Alex R, Muliyil J. Selfharm and self-poisoning in southern India: choice of poisoning agents and treatment. Trop Med Int Health. 2009;14(7):761-5.

2. Chrispal A. Cleistanthus collinus poisoning. J Emerg Trauma Shock. 2012;5(2):160-6.

3. Subrahmanyam DKS, Mooney T, Raveendran R, Zachariah B. A clinical and laboratory profile of Cleistanthus collinus poisoning. J Assoc Physicians India. 2003;51(4):1052-4

4. Eswarappa S, Chakraborty AR, Palatty BU, Vasnaik M. Cleistanthus collinus poisoning: case reports and review of the literature. J Toxicol Clin Toxicol. 2003;41(4):369-72.

5. Kumar MR, Ramaswamy S, Jayanthi M, Raveendran R. Alpha-adrenergic receptor blocking effect of Cleistanthus collinus (Roxb.) Benth. and Hook f. leaf extract on guinea pig isolated smooth muscle preparations. Indian J Exp Biol. $2011 ; 49(4): 339-42$.

6. Pinho PMM, Kijjoa A. Chemical constituents of the plants of the genus Cleistanthus and their biological activity. Phytochem Rev. 2007;6(1):175-82.

7. Parasuraman S, Raveendran R. Effect of cleistanthin A and B on adrenergic and cholinergic receptors. Pharmacogn Mag. 2011;7(27):243-7.

8. Abrahm DR, Hollingsworth PJ, Smith CB, Jim L, Zucker LB, Sobotka PA. Decreased alpha 2-adrenergic receptors on platelet membranes from diabetic patients with autonomic neuropathy and orthostatic hypotension. J Clin Endocrinol Metab. 1986;63(4):906-12.

9. Parasuraman S, Raveendran R, Vijayakumar B, Velmurugan D, Balamurugan S 
Molecular docking and ex vivo pharmacological evaluation of constituents of the leaves of Cleistanthus collinus (Roxb.) (Euphorbiaceae). Indian J Pharmacol. 2012;44(2):197-203.

10. Yun-Choi HS, Park KM, Pyo MK. Epinephrine induced platelet aggregation in rat platelet-rich plasma. Throm Res. 2000;100(6):511-8.

11. Rao AK, Willis J, Kowalska MA. Differential requirements for platelet aggregation and inhibition of adenylate cyclase by epinephrine: studies of a familial platelet alpha 2-adrenergic receptor defect. Blood. 1988;71(2):494-501.

12. Kerry R, Scrutton MC, Wallis RB. Mammalian platelet adrenoceptors. Br J Pharmacol. 1984;81(1):91-102.

13. Berlin I, Crespo-Laumonnier B, Cournot A, Landault C, Aubin F, Legrand J. The $\alpha 2$-adrenergic receptor antagonist yohimbine inhibits epinephrine induced platelet aggregation in healthy subjects. Clin Pharmacol Ther. 1991;49(4):362-9.

14. Požgajova M, Sachs UJH, Hein L, Nieswandt B. Reduced thrombus stability in mice lacking the $\alpha_{2 A}$ adrenergic receptor. Blood. 2006;108(2):510-4.

15. Berlan $M$, Verge RL, Galitzky J, Corre PL. $\alpha_{2}$-adrenoceptor antagonist po- tencies of two hydroxylated metabolites of yohimbine. $\mathrm{Br} J$ Pharmacol. 1993; 108(4):927-32.

16. Savontaus E, Fagerholm V, Rahkonen O, Scheinin M. Reduced blood glucose levels, increased insulin levels and improved glucose tolerance in $\alpha 2 \mathrm{~A}$ adrenoceptor knockout mice. Eur J Pharmacol. 2008;578(2):359-64.

17. Rosengren AH, Jokubka R, Tojjar D, Granhall C, Hansson O, Li DQ. Overexpression Of Alpha 2A-Adrenergic Receptors Contributes To Type 2 Diabetes. Science. 2010;327(5962):217-20.

18. Langer J, Panten U, Zielmann S. Effects of $\alpha$-adrenoceptor antagonists on clonidine induced inhibition of insulin secretion by isolated pancreatic islets. $\mathrm{Br}$ J Pharmac. 1983;79(2):415-20.

19. Hirose $H$, Maruyama $H$, Ito K, Kido K, Koyama K, Saruta T. Effects of $\alpha_{2}$ and $\beta$-adrenergic agonism on glucagon secretion from perfused pancreata of normal and streptozocin-induced diabetic rats. Metabolism. 1993;42(8):1072-6.

20. Minamidate $A$. The effect of stimulation of alpha and beta-adrenergic receptors in the isolated rat jejunum. Jpn J Pharmacol. 1971;21(4):439-46. 